\title{
Analysis of Sticky Cotton by Near-Infrared Spectroscopy
}

\author{
F. E. BARTON II,* J. D. BARGERON III, G. R. GAMBLE, D. L. MCALISTER, and \\ E. HEQUET \\ USDA-Agricultural Research Service, Richard B. Russell Agricultural Research Center, P.O. Box 5677, Athens, Georgia 30604. \\ 567 (F.E.B.); USDA-Agricultural Research Service, Cotton Quality Research Station, Ravenel Center Place, Clemson, South \\ Carolina 29631 (J.D.B., G.R.G., D.L.M.); and International Textile Center, P.O. Box 5888, Lubbock, Texas 79408-5888 (E.H.)
}

\begin{abstract}
"Stickiness" in cotton is a major problem affecting throughput in cotton gins and spinning mills alike. Stickiness is thought to be caused by the deposition of sugars by insects, principally aphid and whitefly, on the open boll. Fourier transform near-infrared (FTNIR) spectroscopy was used to develop models for sugar content from high-pressure liquid chromatography (HPLC), thermodetector, and mini-card data. A total of 457 cotton samples were selected to represent both Upland and Pima varieties and cotton processing before and after ginning. The Unscrambler was used to develop the models. A successful model was made to determine the mini-card value and successfully detect "stickiness". The standard error of cross-validation (SECv) was 0.26 with an $R^{2}$ of 0.96 . The model was not improved by increasing the range of "stickiness" as measured by the mini-card from the usual $0-3$ scale to a scale of $0-8$. If a value is determined to be greater than 1 it will be difficult to blend bales at a spinning plant "opening line" to allow for maximum efficiency of spinning.
\end{abstract}

Index Headings: Near-infrared spectroscopy; Cotton; Stickiness.

\section{INTRODUCTION}

Sticky cotton is a world-wide problem for the textile industry and has been for some years. ${ }^{1.2}$ The "stickiness" results from insect-deposited sugars, principally from whitefly and aphids, and from plant sugars from immature fibers. The insect sugars present the greatest problem as they build up very quickly on rollers and saws in both types of cotton gins and the rotors in the spinning plant. The cost of controlling the insects that deposit the sugars is very large, $\$ 200000000.00$ over the $1994-1998$ seasons in Texas, Arizona, and California. ${ }^{3}$ Solutions to the problem such as insect control have been marginally successful. The insects do not harm the crop and by the time an insect problem is visible the sugars have already been deposited. To spray without obvious need to control insects is expensive and environmentally unsound. Cleaning the cotton with water and enzyme preparations has been somewhat successful in removing the surface-deposited sugars, as have bacterial preparations, but again these measures are costly and are processes that interfere with normal cotton trade. ${ }^{4}$ The most definitive works on the composition and individual properties of the isolated sugars are those of Hendrix (1999, and references cited therein). ${ }^{5-11}$ These measurements of individual sugars have not correlated well with any empirical measurement of stickiness and the mini-card is not precise enough to produce reliable indications. Attempts to correlate to the near-infrared spectra of cottons with lower resolution dispersive instruments have not been accurate enough to de-

\footnotetext{
Received 18 March 2005; accepted 26 August 2005.

* Author to whom correspondence should be sent.
}

tect marginally sticky samples. A new spectroscopic approach is required that allows the sensor to be optimized for the small molecular weight sugars (MW 500) such that the molar concentration will allow them to be seen above the spectrum of the polymeric cellulose (MW > 150000 ). One approach would be to use an interferometer with a resolution of $4 \mathrm{~cm}^{-1}$. The big issue is what to use for a reference method.

There are five reference methods for measuring sticky cotton: ${ }^{2}$ (1) $\mathrm{pH}$ spray indicator test, (2) Benedict's test for reducing sugars, (3) USDA potassium ferricyanide test, (4) mini-card, and (5) thermodetector. Additionally, there are high-pressure liquid chromatography (HPLC) and mass spectrometer methods that can be used to separate, identify, and quantify simple sugars as well. ${ }^{5}$ These methods all suffer from one or more deficiencies that make them ineffective for use in the industry. The $\mathrm{pH}$ spray, Benedict's, and ferricyanide tests are primarily qualitative, with some indication by color variation as to the amount of reducing sugar present. Further, reducing sugar concentration does not correlate well with stickiness. The mini-card gives a good correlation with stickiness, but the precision is not acceptable. It also suffers from the same problem that faces the industry, i.e., it requires extensive cleaning of the card after use, making it a very slow procedure. The thermodetector is fast enough, but it is not as reliable or reproducible as one would like and is very expensive. The mass spectrometer and HPLC methods are very precise but are slow and expensive as currently employed. The industry needs a reliable and inexpensive test that can be used in-line to alert them to a module or bale of sticky cotton so that remedial steps can be taken before it enters the normal processing. Nearinfrared spectroscopy (NIRS) will be used to develop a method to sense stickiness and to measure the sugars present.

\section{MATERIALS AND METHODS}

Samples. A total of 457 samples were collected over a three-year period. Samples represented both "Upland" and "Pima" cotton varieties. The cottons were collected by the Cotton Quality Research Station, Clemson, South Carolina, as part of ongoing spinning studies. All samples were taken through a mini-card at the Clemson, South Carolina, Cotton Quality Research Station. The values of $0,1,2$, or 3 are assigned to the cotton based on how the cotton behaves as it comes over the lapping roller.

Reference Data Procedures. Mini-card. The minicard (Fig. 1) is a small (6 ft long, by $4 \mathrm{ft}$ high, by $2 \mathrm{ft}$ wide, $1500 \mathrm{lbs}$ ) version of the piece of textile equipment known as an opening card. The opening card is a device 


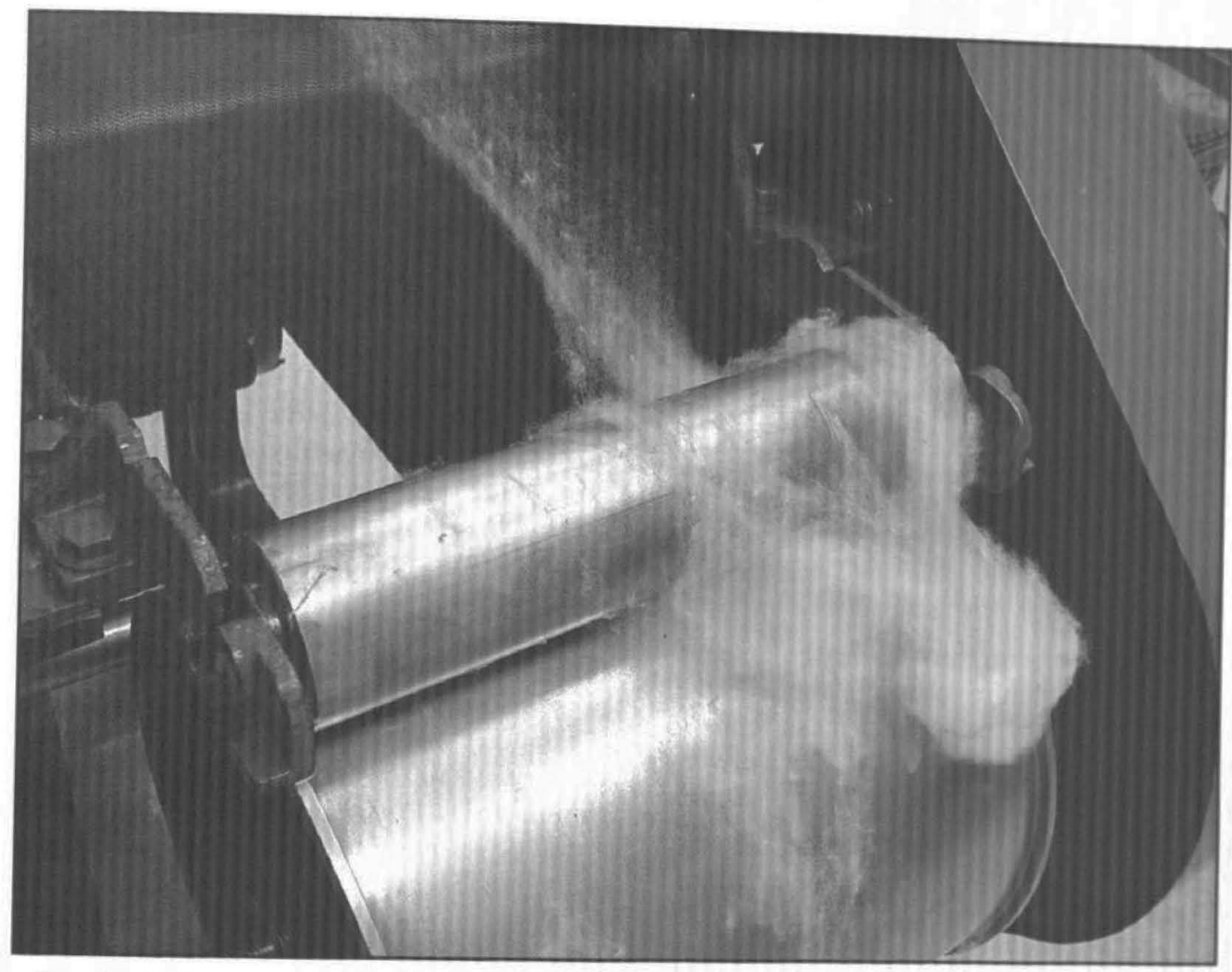

Fig. 1. Cotton fiber lapping the rollers on the mini-card. This sticky cotton would be scored as a 2.0.

for taking the raw cotton from the bale and opening the fiber so that a fine mat is formed, which is subsequently spun into a coarse tube of cotton. The opening card is a large machine that could be placed in a $14 \times 14$ foot $(5$ $\times 5$ meter) room. In spinning mills there would be many of these devices in parallel lines, and in large mills, over 50 of them. These machines are the first step in spinning cotton to thread. In Fig. 1 this fine mat/web of fiber can be seen as it comes out and is to pass between the small and large roller. Clean cotton passes through and is then twisted into the coarse cotton strand called a "sliver". Below is a description of how a sample is prepared for the Mini-Card and how the results are judged. The results are objective only with a well-trained operator.

Material. Raw cotton only (cotton that has been ginned and baled, but no other treatment).

Sample Size. Ten (10) grams. It is important that this 10 grams be formed into a uniform, neat, approximately 4.25 -in. wide $\times 9.75$-in. long $(11 \mathrm{~cm}$ wide $\times 25 \mathrm{~cm}$ long $)$ narrow, rectangular-shaped sample.

Test Procedure. Feed the 4 -in. wide mat between the input feed rolls of the mini-card as evenly as possible. When the cotton reaches the output, place the cotton web between the delivery rolls. Count and remove each web wrap until all of the 10-gram sample has passed through the mini-card. After each sample, clean the delivery rolls with tap water and paper towels until dry and all sticky residue is removed from both rolls. (Note: If two consecutive samples receive a rating of 3 , indicating a level of "Heavy Stickiness", the mini-card must be purged by running a clean, non-sticky 10-gram sample through the machine).

Following are five factors to be considered in developing descriptive terms for the mini-card stickiness rat- ings: (1) Number of times stickiness occurred (most important factor). (2) Size of the sticky masses, (3) Tendency of the fiber web to wrap around the delivery rolls. (4) Time for stickiness to develop and intensify. (5) Amount of sticky residue and fiber mass remaining on the delivery rolls after the test.

0, No Stickiness. This rating is self explanatory and basically means that the test sample did not stick to the delivery rolls during passage through the mini-card. However, experience dictates that very often a few specks of stickiness will occur on the delivery rolls (even for cottons that are obviously not honeydew contaminated). These specks nay be small leaf particles, other trash, neps (hard spheres of fiber which can be spun into the thread and weaken it), etc., that will not cause stickiness in the yarn manufacturing processes. (These are the materials that during mill carding will adhere to the crush rolls, but will be removed by the scraper blade without affecting production or quality). Therefore, a sample that exhibits less than 5 specks of stickiness and such specks make no more than 1 revolution on the delivery rolls may still be rated as having no stickiness.

1, Light Stickiness. Sample sticks to delivery rolls between 5 and 15 times during passage through the minicard. Most of the specks of stickiness are small and make only one revolution on the delivery rolls. There is little tendency for the web to wrap around the delivery rolls. The stickiness nay increase slightly during the progress of the test. The delivery rolls may contain a few specks of sticky residue of various sizes or a few fibers in spots after completion of the test.

2, Moderate Stickiness. Sample sticks to delivery rolls between 16 and 25 times during passage through the mini-card. The specks of stickiness may vary in size and 


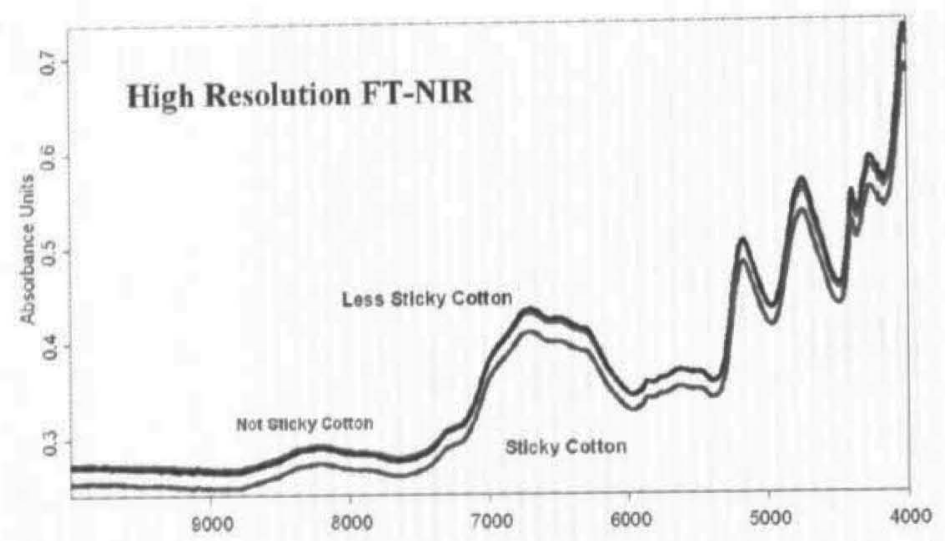

FIG. 2. FT-NIR spectra of sticky versus less and not sticky cotton at $4 \mathrm{~cm}^{-1}$ resolution.

often make several revolutions on the delivery rolls, causing the web to stick during each revolution. The web will often wrap around the delivery rolls. Stickiness tends to become more severe as the test progresses. The delivery rolls may contain some sticky residue and fiber mass after completion of the test.

3, Heavy Stickiness. Sample sticks to delivery rolls 26 times or more during passage through the mini-card. Both small and large masses or large numbers of small masses of stickiness appear on the delivery rolls and remain for many revolutions. Stickiness begins immediately after start of the test and becomes more and more severe as the test progresses because of the heavy residue and lint accumulation on the delivery rolls. It is very difficult to keep the web from wrapping around the delivery rolls. Heavy sticky residue and fiber mass may remain on the delivery rolls after the test.

High-Pressure Liquid Chromatography. A 1 g sample of each cotton was extracted with $20 \mathrm{~mL}$ deionized water. An aliquot of $0.5 \mathrm{~mL}$ was analyzed for individual sugars using high-performance anion exchange chromatography performed on a Dionex DX-500 using pulsed amperometric detection under conditions similar to those described previously. ${ }^{5}$ Briefly, two Dionex Carbopac PA$1(4 \times 250 \mathrm{~mm})$ columns were connected in series and elution was carried out at $0.75 \mathrm{~mL} / \mathrm{min}$ using $200 \mathrm{mM}$ $\mathrm{NaOH}$ as the mobile phase and a sigmoidal gradient of 0 to $500 \mathrm{mM} \mathrm{NaOAc}$. The $0.5 \mathrm{~mL}$ aliquot was analyzed either directly or following a $10 \times$ dilution in order to keep individual sugar concentrations below $100 \mathrm{mg} / \mathrm{L}$, above which saturation of the amperometric detector begins to occur.

Near-Infrared Spectroscopy. Near-infrared spectra were obtained on a Bruker model FT22-N, (Bruker Optics, Billerica, MA) in reflectance mode equipped with an integrating sphere and $\mathrm{PbS}$ detector over a frequency range of $10000-4000 \mathrm{~cm}^{-1}$ at $4 \mathrm{~cm}^{-1}$ (3112 data points). The instrument was equipped with a large sample cup $(89 \mathrm{~mm})$. The cotton was placed in the cup and the gold standard placed on top to put even pressure on the mat. The amount of cotton was approximately $15 \mathrm{~g}$. After the sample was scanned the cotton bat was rearranged and the spectra retaken. The samples were rearranged a total of three times each and the spectra averaged at the end. The data were collected on a PC with Bruker OPUS software and transferred to Unscrambler, ver. 7.6 (CAMO,
TABLE I. Standard error of cross-validation (SECv) for the original data set.

\begin{tabular}{llcc}
\hline \multicolumn{1}{c}{ Constituent } & \multicolumn{1}{c}{ Range } & $R^{2}$ & SECv \\
\hline Mini-Card & $0-3$ & 0.47 & 2.5 \\
Glucose & $7-1300 \mathrm{ppm}$ & 0.90 & 5.20 \\
Trehalulose & $0-4000 \mathrm{ppm}$ & 0.95 & 43.1 \\
Melezitose & $0-2000 \mathrm{ppm}$ & 0.91 & 20.0 \\
Thermodectector & $1-90$ & 0.87 & 12.1 \\
\hline
\end{tabular}

Throndheim, Norway) for model development. Dispersive spectra were obtained on a Foss NIRSystem's (Silver Spring, MD) 6500 with a silicon visible detector and $\mathrm{PbS}$ NIR detector from 400-2500 nm (1050 datapoints) at 2 $\mathrm{nm}$ interval and $10 \mathrm{~nm}$ bandpass (resolution).

Chemometrics. Calibrations were developed using the PLS1 (partial least squares) and Marten's Regression programs in the Unscrambler software package vers. 7.6 (CAMO, Trondheim, Norway). The global H (Mahalanobis distance) for outlier detection of spectra was set at 2.5 , and $\mathrm{T}$ for reference data was 2.5 as well. Data for the Foss 6500 was acquired with the Infrasoft International (ISI) (Port Matilda, PA) NIRS3 package. Models were developed in NIRS3 or exported to Unscrambler with the ISI file converter.

\section{RESULTS AND DISCUSSION}

To develop an NIRS method for "stickiness" in cotton, a number of problems need to be alleviated. The first problem is to determine the spectral resolution needed to yield a satisfactory correlation since we will be trying to find small sugars, monomers, dimmers, or trimers against a cellulose matrix. Early attempts with the dispersive instruments at $10 \mathrm{~nm}$ bandpass $\left(20 \mathrm{~cm}^{-1}\right.$ resolution) gave indications that stickiness could be modeled, but the results were unsatisfactory. ${ }^{12}$ Preliminary data with spectra from an FT-NIR at $4 \mathrm{~cm}^{-1}$ have been more successful (Fig. 2). There is evidence of the appearance of the sharper bands associated with the smaller carbohydrates on top of the cellulose spectra in Fig. 2. Thus, it appears that 4 $\mathrm{cm}^{-1}$ resolution is needed to make the measurement.

The second problem is to determine that the reference value truly correlates to stickiness. Preliminary data shows that good correlations can be obtained for all measures of reducing sugar and a reasonable one for the minicard (Table I). However, the sugars do not correlate well to stickiness. Good models for the amount of reducing sugar, glucose, melezitose, and trehalose can be developed. The standard error of cross-validation (SECv) and $R^{2}$ are given in Table I. While the high $R^{2}$ and relatively low SECv would seem adequate to predict the sugars content, they are not adequate to predict stickiness. Stickiness is more than just the simple sugars deposited by the insects. The sugar spots are small on the surface of the boll and selecting a representative sample is difficult. The sugar assays require extraction and subsequent analysis of a small sample by HPLC. While the precision of the HPLC is excellent, the results are quite variable for repeat samples, and thus the accuracy of the method is not ideal for NIR method development. In the end, while the reference assay for sugars may be precise and repeatability adequate, what the instrument "sees" as a sample given the spatial geometry of the sugar deposition patterns may 
TABLE II. Standard error of cross-validation (SECv) for the original data set and the enlarged data set on the FT-NIR spectrometer.

\begin{tabular}{lccl}
\hline \multicolumn{1}{c}{ Data set } & Number of samples & \multicolumn{1}{c}{$R^{2}$} & $\mathrm{SECv}$ \\
\hline Original & 51 & 0.47 & 2.5 \\
Enlarged (MSC/MC) & 225 (out of 300) & 0.87 & 1.50 \\
Enlarged (2 (nd $^{\text {derivative) }}$ & 298 (out of 300) & 0.96 & 0.25 \\
\hline
\end{tabular}

be quite different. Therefore, a large population of samples is required to select those spectra and reference analyses that match.

The mini-card has the opposite problem. The mini-card is an accurate determination of stickiness as it is perceived by the operator of the textile processing equipment, but the scale is narrow, with values of $0,1,2$, and 3 , and very poor precision $(+/-1)$. A preliminary model based on 51 samples (SECv 0.99, $R^{2} 0.93$, with 8 PLS factors; SECv $2.5, R^{2} 0.47$, with 3 PLS factors) correctly identified the sticky samples in a small ( 7 samples: 5 sticky, 1 light, and 1 clean) second set of cotton samples. However, the standard error of prediction (SEP) was too large to be a valid measure and a much larger set of reference samples is required. The samples were all processed through the cotton gin and had been subjected to heat and were not truly representative of samples arriving at the cotton gin. However, it was a sufficiently definitive result to be considered "proof-of-concept".

A third problem was the development of the needed database of spectra and reference data. Stickiness is an intermittent problem and finding good samples took two years and the analysis another year. A set of 300 cotton samples representing location, cultivar, and level and type of stickiness was assembled out of the 457 total samples, which was deemed to be an appropriate number for the method. The samples included representatives of both cultivar and location that are not sticky, sticky with silverleaf whitefly and aphid deposits, sticky with each insect's honeydew deposits separately, with plant sugars, and then varying degrees of stickiness. The laboratory analyses for sugars and stickiness were conducted by the ARS Cotton Quality Research Station, Clemson, South Carolina.

Reflectance mode was used since the insect-deposited sugars are a surface phenomena.

The fourth problem will be to develop classification models that will be set up to place samples in the sticky/ not sticky categories. Many times these models have data space that overlaps, creating areas of indeterminate classification, and if so then other classes of moderate stickiness must be invoked until classification can be achieved.

Table II contains the SECv and $R^{2}$ for the initial data set of 51 samples. This set gave a model that could be used as stated above, but the data as shown in Table I suggests that an $R^{2}$ of 0.47 is not very good. The spectra of the second set of samples (300) were subjected to mathematical pretreatment of multiplicative scatter correction (MSC) and mean centering followed by PLS1 model development. These models were satisfactory but very poor predictors. Many samples were identified as outliers. It is reasonable to expect a large number of outliers in this set as the sample seen by the FT-NIR may

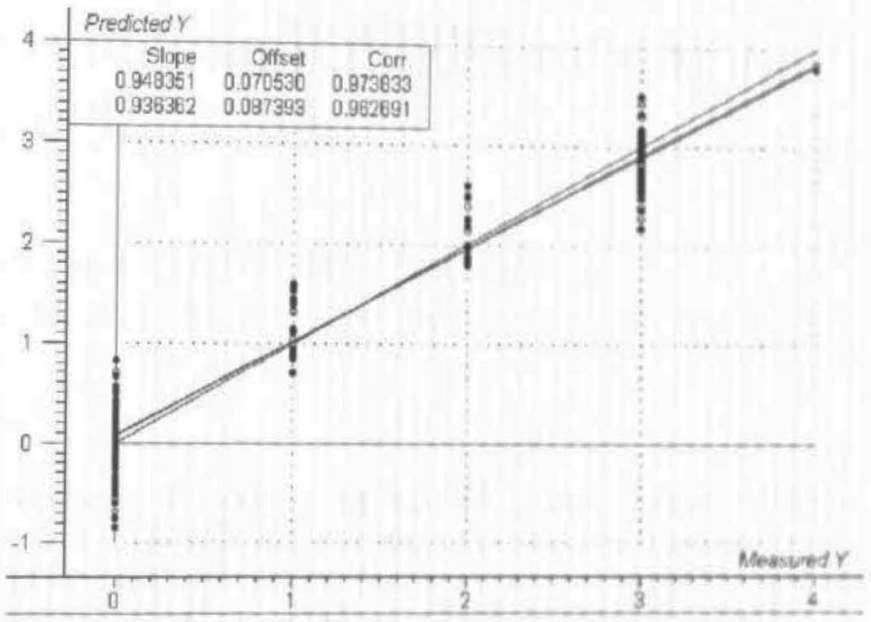

FIG. 3. A scatter plot of predicted $(\mathrm{Y})$ versus reference $(\mathrm{X})$ values for stickiness in cotton; the top line values are for calibration, while the second line values are the prediction from which the SECV is calculated.

not be the one seen by the mini-card. The raw cotton is introduced to the mini-card (Fig. 1) and fed through roller combs, which open the fiber and produce a fine web. The web is fed through two smooth rollers, a large diameter lower one and a smaller diameter upper lapping roller. Stickiness is determined by the operator based on how badly the cotton web "laps" over and sticks to the upper roller. The determination of the integral value of $0,1,2$, or 3 is assigned based on the criteria outlined in the Experimental section. This is not a precise measurement that can be easily modeled by spectroscopy. The model developed in Table II, row 2, looked adequate but did not perform well on a separate validation set. A large negative bias (10.5) was observed. Figure 2 shows an "albedo" difference for clean and sticky samples, with the sticky samples being more reflective. A second model was developed without either the MSC or mean centering to allow the reflective offset information to be used. An 11-point second-derivative gave the best model as shown in Table II, row 3 . This model contained very few outliers and the actual versus predicted plot in Fig. 3 has a slope

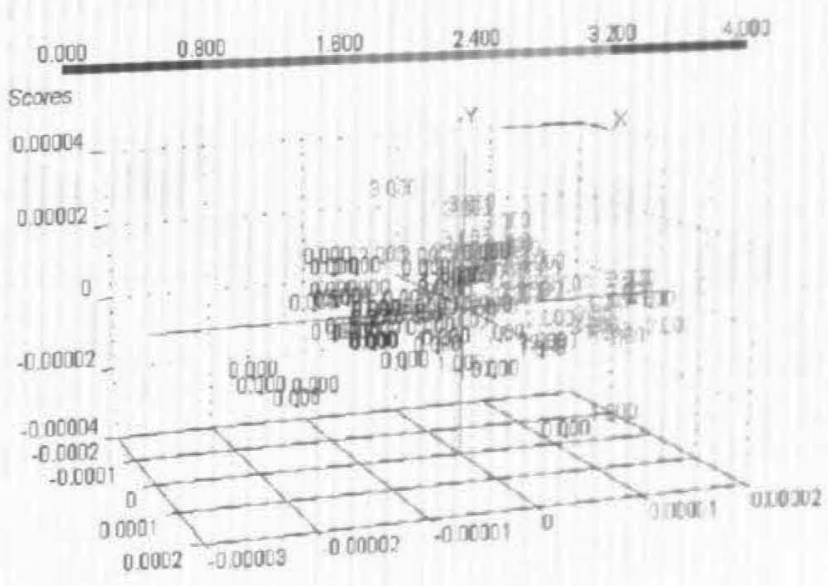

A plot of the first three PLS scores. Data points on the left a's, while the ones on the right of the axis are 1 's. 2 s. and 3's. 


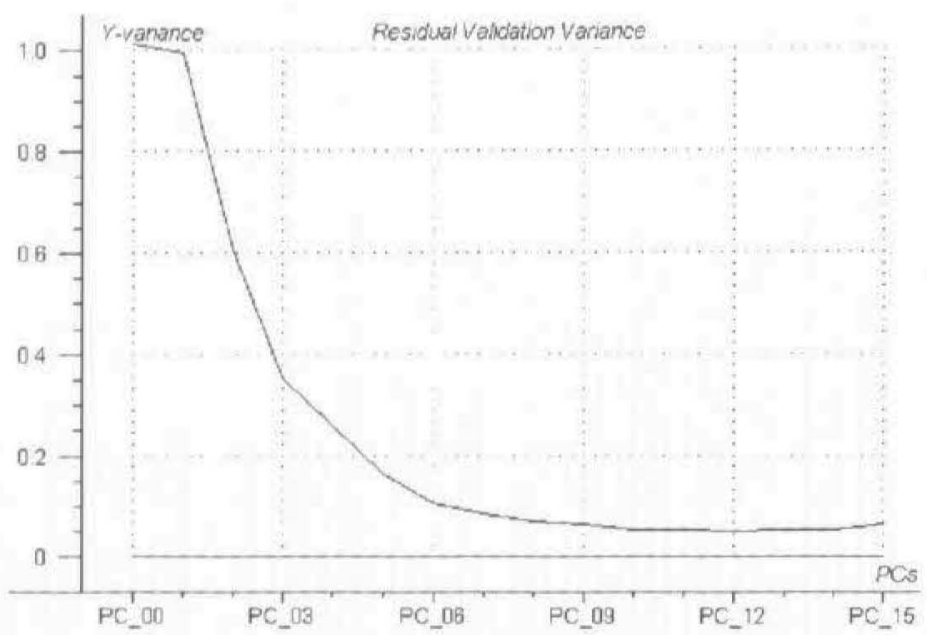

Fig. 5. Plot of the SECv (Y) versus number of PLS factors. The steep slope indicates the significance of the first three PLS factors.

near 1.0. This model cleanly separated the cottons by their stickiness scores as shown in the three-dimensional score plot in Fig. 4. The sticky samples are on the right side and the clean samples on the left. These first three factors were the most important, as can be seen in Fig. 5 from the steepness of the drop in SECv with the addition of each factor. This model has a small SECv $(0.25)$ and correctly predicted a validation set of 23 samples from a different crop year with an SEP of 0.5 and an $R^{2}$ of 0.81 (Fig. 6).

The set of 300 samples was scanned in a dispersive instrument at $10 \mathrm{~nm}$ bandpass (resolution). This compares to a resolution of $90 \mathrm{~cm}^{-1}$ at $1000 \mathrm{~cm}^{-1}(1000 \mathrm{~nm})$ and $16 \mathrm{~cm}^{-1}$ at $4000 \mathrm{~cm}^{-1}(2500 \mathrm{~nm})$. The results are shown in Table III, where the $R^{2}$ was 0.68 and the SECv was 2.90. This is insufficient to determine stickiness. The results just using the visible portion were much better $\left(R^{2}\right.$ of 0.88 and SECv of 0.56 ), twice the error of the FT instrument, an indication that the albedo difference (see Fig. 2) can be related directly to stickiness.

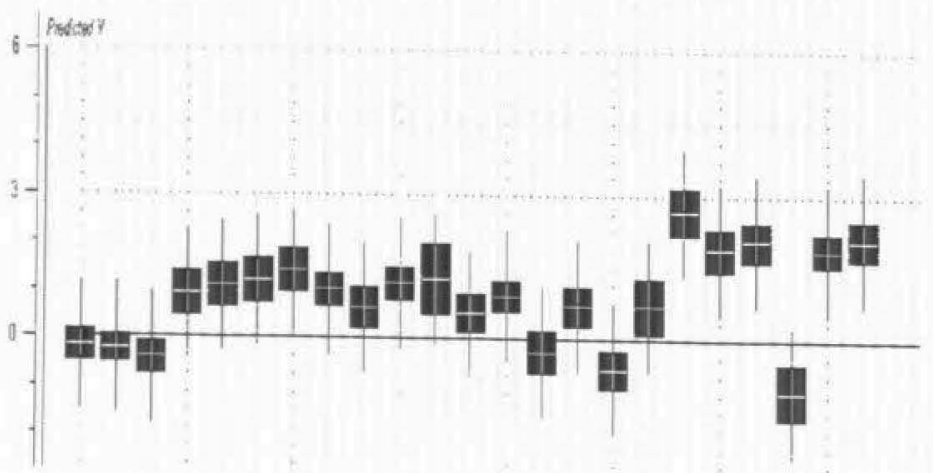

Fig. 6. Plot of 23 predicted samples. The 0's and 1's are separated from the 2's and 3 's.
TABLE III. Standard error of cross-validation (SECv) for the enlarged data set on the dispersive instrument.

\begin{tabular}{lccc}
\hline $\begin{array}{l}\text { Instrument } \\
\text { spectral range }\end{array}$ & Number of samples & $R^{2}$ & SECv \\
\hline VIS and NIR & 127 (out of 300) & 0.68 & 2.90 \\
VIS only & 154 (out of 300) & 0.88 & 0.56 \\
\hline
\end{tabular}

\section{CONCLUSION}

First, FT-NIR can be used to detect stickiness in raw cotton. Second, the poor performance of the sugar models indicates that something other than simple sugars is important to model stickiness. It may be larger oligimers of the sugars and/or proteins secreted with the sugars.

The level of model development described in this study is necessary because we intend to evaluate a number of sensors that can be used on-line for the stickiness application. There are several process FT-NIR instruments commercially available and subsequent studies will determine their suitability and placement in the cotton ginning process. A digital array scanning interferometer (DASI) has been built and will be used in future studies. These instruments have never been tested for calibration transfer or their suitability for use in a multi-instrument network.

1. P. J. Wakelyn, P. K. Adair, N. R. Bertonniere, C. K. Bragg, and H. B. Poole, Focus on Cotton Textile Research: Results Report (Agri Business Group, Indianapolis, IN, 1998).

2. D. E. Brushwood and H. H. Perkins, Canadian Tex. J. Sept., 54 (1993).

3. P. C. Ellsworth, R. Trondstad, J. Lester, P. B. Goodwell, L. D. Godfrey, T. J. Henneberry, D. Hendrix, D. Brushwood, S. E. Naranjo, S. Castle, and R. L. Nichols, Sticky Cotton Sources and Solutions (The University of Arizona Cooperative Extension, IPM Series No. 13, AZ1156-12, 1999).

4. W. Coates, Determination of a field method to apply enzyme to sticky cotton, Final report of Cotton Incorporated project 94:1202 (1996).

5. D. L. Hendrix, Proc. Beltwide Cotton Conferences 1, 47 (1999).

6. D. L. Hendrix, B. Blackledge, and H. H. Perkins, Jr., "Development of methods for the detection and elimination of insect honeydews on cotton fiber", in Proceedings, Beltwide Cotton Research Conference (National Cotton Council, Memphis, TN, 1993), pp. 16001602.

7. D. L. Hendrix, T. J. Henneberry, T. Steele, C. C. Chu, and W. Coates, "Laboratory simulation of cotton modules", in Proceedings, Beltwide Cotton Research Conference (National Cotton Council, Memphis, TN, 1997).

8. D. L. Hendrix and H. H. Perkins, Jr., "Detection of insect honeydew on cotton lint and elimination of honeydew stickiness by enzyme treatment", in Proceedings, Beltwide Cotton Research Conference (National Cotton Council, Memphis, TN, 1995), pp. 3, 4.

9. D. L. Hendrix, T. L. Steele, and H. H. Perkins, Jr., "Bemisia honeydew and sticky cotton", in Bemisia 1995: Taxonomy, Biology, Damage and Management, D. Gerling and R. T. Mayer, Eds. (Intercept, Andover, 1996), pp.189-199.

10. D. L. Hendrix and Y. A. Wei, "Detection and elimination of honeydew excreted by the sweetpotato whitefly feeding upon cotton", in Proceedings, Beltwide Cotton Research Conference (National Cotton Council, Memphis, TN, 1992), pp. 671-673.

11. D. L. Hendrix and Y. A. Wei, Carbohydrate Res. 25, 329 (1994).

12. D. E. Brushwood and Y. J. Han, J. Cotton Sci. 4, 137 (2000). 